\title{
Effectiveness of Inactivated Influenza Vaccine Among Nursing Home Residents During an Influenza Type A (H3N2) Epidemic
}

\author{
Suzanne E. Obmit, DrPH, Nancy H. Arden, MN, ${ }^{+}$and Arnold S. Monto, MD*
}

OBJECTIVES: To evaluate the use of influenza vaccine in nursing homes and its effectiveness in reducing the likelihood of influenza-like illness.

DESIGN: A retrospective case-control study with active identification of influenza infection.

SETTING: All nursing homes in a seven-county study area in southern lower Michigan were eligible for participation. Analyses were based on data collected from 23 homes with documented influenza transmission.

PARTICIPANTS: Persons aged 65 years or older who were residents of the nursing homes under study during the influenza type $A(H 3 N 2)$ outbreak in 1989-1990.

MEASUREMENTS: Residents were identified as cases or controls based on occurrence of febrile respiratory illness meeting a case definition. Demographic and underlying illness information were gathered as were data on the use of influenza vaccine, antibiotics, and antivirals. Characteristics of the nursing homes were also recorded. Logistic regression analyses were carried out to determine vaccine effectiveness.

MAIN RESULTS: Determinants of vaccine use were different from those observed in a parallel community-based study. In a multivariate model that considered the effects of resident and nursing home characteristics, vaccinated residents were significantly less likely than those who were not vaccinated to have an influenza-like illness (OR $=.58(95 \% \mathrm{CI}, .43-.78)$, $P<.001$, imputed vaccine effectiveness estimate of $42 \%)$. Vaccination was more effective in younger residents (those aged 65 to 84 ) then in older residents (those older than 84 years).

CONCLUSIONS: Influenza vaccination was effective in reducing the likelihood of influenza-like illness in nursing home residents. Effectiveness appeared to be related to age, which may function as a surrogate for related immunologic factors. Older nursing home residents should be targeted for newer vaccines and/or potential prophylactic use of antivirals. J Am Geriatr Soc 47:165-171, 1999.

Key words: elderly; influenza; influenza vaccine; nursing homes; vaccine effectiveness

From the "School of Public Health, University of Michigan, Ann Arbor, Michigan; and the †National Vaccine Program, Centers for Discase Control and Prevention, Atlanta, Georgia.

Address correspondence to Arnold S. Monto, MD, School of Public Health, University of Michigan, 109 Observatory, Ann Arbor, MI 48109-2029
Tnactivated influenza vaccines have been in use for more Ithan 50 years. Repeated controlled evaluations of their efficacy in young adults, typically military recruits, demonstrated prevention of laboratory-confirmed clinical disease in the range of 70 to $90 \% .^{1}$ Until recently, many have questioned the value of influenza vaccination in an older, more frail population because of the occurrence of nursing home outbreaks even in situations where the circulating virus was similar to that in the vaccine. ${ }^{2-4} \mathrm{~A}$ series of observational studies have confirmed that, in older persons living in the community, the vaccine has been significantly protective against outcomes such as hospitalization and, in some cases, death. ${ }^{5-8}$ Largely as a result of these studies, influenza vaccination became a Medicare part B-covered benefit in 1993.

The situation in nursing homes may be the most extreme test for the current vaccine since, in addition to the increased possibility for spread of infection in these closed environments, many residents are older than 85 years of age and have multiple underlying conditions. ${ }^{10}$ Most influenza-associated deaths occur among older people, and residents of nursing homes are at especially high risk for severe influenza-related complications. Since the emergence of influenza type $\mathrm{A}(\mathrm{H} 3 \mathrm{~N} 2)$ in 1968 , seasons dominated by this influenza virus subtype have been associated with the highest mortality compared with seasons dominated by influenza type $\mathrm{A}(\mathrm{H} 1 \mathrm{~N} 1)$ or type $\mathrm{B}$ viruses. The outbreak during the 19891990 influenza season was more severe than that of the average $A(H 3 N 2)$ season, with major pneumonia and influenza mortality among older people nationally and increased morbidity in younger individuals. ${ }^{11}$ In an assessment of the impact of influenza epidemics on mortality in the United States from 1972 through 1992, using a severity index ranging from 1 (mildest) to 10 (most severe), the 1989-1990 season was in severity category 6 , one category below the type $\mathrm{A}(\mathrm{H} 3 \mathrm{~N} 2)$ pandemic of $1968-1969 .^{12}$ Influenza $\mathrm{A}(\mathrm{H} 3 \mathrm{~N} 2)$ viruses isolated nationally were antigenically similar to $A / S h a n g h a i / 11 / 87$, the $A(H 3 N 2)$ component of the 1989-1990 influenza vaccine. The current report describes an evaluation that estimated the effectiveness of the vaccine in preventing influenza-like illness in residents of nursing homes experiencing outbreaks of the type $\mathrm{A}(\mathrm{H} 3 \mathrm{~N} 2)$ virus using case-control methodology.

\section{METHODS}

Surveillance

The study area consisted of seven counties in southcentral and southwestern lower Michigan. A community- 
based surveillance system was established within this area from November through April to determine the timing and intensity of influenza virus activity and influenza-like illness. ${ }^{13}$ All 83 nursing homes in the seven-county study area were eligible and encouraged to participate in surveillance by reporting weekly on the number of new cases of influenzalike illness occurring among residents. Influenza-like illness (ILI) was defined as an oral temperature (or rectal or axillary equivalent) of at least $37.8^{\circ} \mathrm{C}$ and one or more of the following signs or symptoms: cough, sore throat, or coryza. ${ }^{14}$ Nursing homes were also asked to submit pharyngeal swab specimens for influenza isolation from residents with influenza-like illness; however, influenza virus isolation was not required to meet the case definition.

After the period of influenza surveillance ended, the weekly incidence of influenza-like illness activity within each participating nursing home was evaluated. Homes that experienced outbreaks $(10 \%$ or more of the resident population developed ILI within a 7-day period during periods of laboratory-confirmed community influenza activity) and homes with definitive influenza introduction, based on influenza virus isolation from pharyngeal swab specimens, were targeted for enrollment in the vaccine effectiveness study. All homes recruited for the effectiveness study were required to have kept patient-specific logs throughout the surveillance period on those residents who met the case definition for ILI.

\section{Study Population}

The eligible study population was comprised of all persons aged 65 years and older who were residents of participating nursing homes, as determined from census lists, at the beginning of the surveillance period, November 1, 1989. All homes conducted influenza vaccination programs from late September through early November 1989, and residents' vaccination statuses resulting from these programs were considered here. Cases were those residents who developed influenza-like illness during the period of laboratoryconfirmed community influenza activity. Controls were drawn from the same study population and were required to have not developed ILI during the study period and to reside in the same facility as the case. Up to three controls were identified and group matched by 5 -year age category to each identified case. Preference was given to selecting controls who resided in the same geographic area of the facility (wing or floor) as the case. Controls who died before the end of the surveillance period and controls who received amantadine were excluded, and, thus, selected controls had the opportunity to become cases throughout the surveillance period.

\section{Data Collection}

Data on cases and controls were collected retrospectively by nursing home staff using survey forms developed by study personnel. Data sources within nursing homes were individual resident medical records, nurses' notes, pharmacy records, infection control logs, and the resident-specific case identification logs generated throughout the surveillance period. For each case or control, information about the following variables was requested: resident's gender, date of birth, admission date, date of onset of illness for cases, 1989-1990 influenza vaccination status, pneumococcal vaccination status, the existence of certain chronic or comorbid conditions (heart disease, lung/pulmonary disease, diabetes, renal disease, cancer, dementia, Parkinson's disease, hypothyroidism, and anemia), assessment of competency to give consent for their own medical treatment, amantadine use, antibiotic use, discharge status and date, and date of death, if applicable.

\section{Data Analysis}

Individuals were classified as vaccinated if their records indicated influenza vaccination for the 1989-1990 season during fall vaccination programs. Those whose records indicated not being vaccinated and those with unknown vaccination status were coded as unvaccinated. Age was represented by six 5 -year categories from 65 to 90 or older. Pneumococcal vaccination status was classified as vaccinated, unvaccinated, or unknown. Specified chronic or comorbid conditions, as described previously, and competency to give consent for their own medical treatment were coded as present if reported as such, absent otherwise. Individuals who were discharged, transferred and/or who died were identified. Cases who died within 1 month of influenza-like illness onset were considered to have died as a result of influenza-related complications. Antibiotic and amantadine use were coded as present if reported as such, absent otherwise.

In order to consider characteristics of individual nursing homes that might affect both the introduction of influenza into the home and transmission of influenza within the home, values for size of home (number of beds) and overall influenza vaccination level (percent vaccinated) for each participating nursing home were obtained from the 1989-1990 Nursing Home Survey ${ }^{15}$ and included in this analysis. Number of beds was expressed as a dichotomous variable representing small ( $\leq 100$ beds) and large (>100 beds) homes, based on a frequency distribution of the number of beds. Percent vaccinated was expressed as a dichotomous variable representing those homes above and below the overall median (and mean) of $75 \%$ vaccinated.

The statistical significance of differences in characteristics between cases and controls and between vaccinated and unvaccinated individuals were evaluated using cross tabulations and chi-square statistics. ${ }^{16}$ Logistic regression analy$\operatorname{ses}^{17}$ were used to estimate the effectiveness of influenza vaccination in reducing the likelihood of influenza-like illness while adjusting for study population and nursing home characteristics that influenced this relationship. Two techniques were used to control for the effect of individual nursing home characteristics on the evaluation of influenza vaccine effectiveness. The first technique used conditional logistic regression to evaluate vaccine effectiveness conditional on nursing home. In this analysis, cases were compared only with controls within the same nursing home, and an overall measure of vaccine effectiveness was estimated. The second technique was developed for the purpose of considering the impact of theoretically important characteristics of nursing homes while estimating overall influenza vaccine effectiveness. Information on number of beds and overall percentage of influenza-vaccinated residents, as described previously, were selected to represent important features of nursing homes. Information on overall percentage of residents vaccinated was not available for five of the nursing homes represented in the case-control study.

Estimates of the effectiveness of vaccine in preventing a rare condition in case-control studies can be derived from the logistic regression models as being equivalent to 1 minus the odds ratio (1-OR). Influenza-like illness in the present study was a relatively frequent outcome, suggesting that this deri- 
vation may not be directly appropriate. In order to permit comparison with the effectiveness measures derived in other studics, calculations that will be referred to as "imputed vaccine effectiveness estimates" are included here in addition to measures of reduced odds or likelihood.

\section{RESULTS}

\section{Influenza Virus Activity}

Influenza $\mathrm{A}(\mathrm{H} 3 \mathrm{~N} 2)$ was the only type of influenza virus detected in the study area during the 1989-1990 season, and the strain was antigenically similar to the strain that circulated nationally and included as the $\mathrm{A}(\mathrm{H} 3 \mathrm{~N} 2)$ component of the 1989-1990 vaccine. Influenza was first isolated from a community-acquired specimen in late November 1989; virus isolation continued through March $1990 .{ }^{13}$ The majority of the late season isolates were from nursing homes. Sixty-five $(78 \%)$ study area homes participated in influenza-like illness surveillance by submitting weekly data on illness incidence. Thirty-one $(37 \%)$ homes provided pharyngeal swab specimens for influenza virus isolation.

\section{Population Characteristics}

Because the underlying assumption of these analyses was that all included cases and controls were exposed to circulating influenza virus, only homes with definitive introduction of influenza were included. Twenty-three nursing homes had evidence of influenza introduction based on outbreak activity (17 homes) or isolation of influenza virus from pharyngeal swab specimens ( 21 homes). ${ }^{15}$ This restriction resulted in a study population of 1198 residents: 361 cases $(30 \%)$ and 837 controls $(70 \%)$.

Table 1 shows the distribution of study population characteristics by case-control status. Overall, $71 \%$ of the study population were reported to have received the influenza vaccine for the $1989-1990$ season; $61 \%$ of cases and $75 \%$ of controls received vaccine $(\mathrm{OR}=.52$ (95\% CI, .40-.67), imputed vaccine effectiveness estimate of $48 \%)$ ). Women, who made up $80 \%$ of the total study population but only $76 \%$ of the cases, were significantly less likely than men to develop ILI (OR $=.74(95 \% \mathrm{CI}, .55-.99))$. Cases and controls did not differ significantly by age categories as determined in the study design. Pneumococcal vaccination confirmed from nursing home records was identified in less than $18 \%$ of the study population and did not differ by case or control status; pneumococcal vaccination status was unknown in $59 \%$ of the study population. Significantly more cases were reported to have lung disease $(O R=1.62(95 \%$ $\mathrm{CI}, 1.05-2.50)$ ) and renal discase (OR $=2.23(95 \% \mathrm{CI}$, 1.14-4.41)), whereas significantly more controls were assessed as being competent to give consent for their own medical treatment $(\mathrm{OR}=.61(95 \% \mathrm{CI}, .45-.82))$.

Table 1. Characteristics of the 1989-1990 Nursing Home Study Population by Case and Control Status. South-central and Southwestern Lower Michigan

\begin{tabular}{|c|c|c|c|}
\hline Characteristic & $\begin{array}{c}\text { Cases } \\
n=361\end{array}$ & $\begin{array}{l}\text { Controls } \\
n=837\end{array}$ & $P$ Value \\
\hline \multicolumn{4}{|l|}{ Gender } \\
\hline Female & $274(75.9)$ & $678(81.0)$ & .045 \\
\hline Male & $87(24.1)$ & $159(19.0)$ & \\
\hline \multicolumn{4}{|l|}{ Age category } \\
\hline $65-69$ & $15(4.2)$ & $52(6.2)$ & NS \\
\hline $70-74$ & $26(7.2)$ & 77 (9.2) & \\
\hline $75-79$ & $51(14.1)$ & $124(14.8)$ & \\
\hline $80-84$ & $89(24.7)$ & $184(22.0)$ & \\
\hline $85-89$ & $89(24.7)$ & $192(22.9)$ & \\
\hline $90+$ & $91(25.2)$ & $208(24.9)$ & \\
\hline \multicolumn{4}{|l|}{ Influenza vaccination 1989-90 } \\
\hline Yes & $220(60.9)$ & $628(75.0)$ & $<.001$ \\
\hline No & $141(39.1)$ & $209(25.0)$ & \\
\hline \multicolumn{4}{|l|}{ Pneumococcal vaccination } \\
\hline Yes & $64(17.7)$ & $144(17.2)$ & NS \\
\hline No & $95(26.3)$ & $188(22.5)$ & \\
\hline Unknown & $202(56.0)$ & $505(60.3)$ & \\
\hline \multicolumn{4}{|c|}{ Comorbid conditions (not mutually exclusive) } \\
\hline Anemia & $22(6.1)$ & $55(6.6)$ & NS \\
\hline Cancer & $12(3.3)$ & $17(2.0)$ & NS \\
\hline Dementia & $162(44.9)$ & $376(44.9)$ & NS \\
\hline Diabetes & $45(12.5)$ & $106(12.7)$ & NS \\
\hline Heart disease & $212(58.7)$ & $455(54.4)$ & NS \\
\hline Hypothyroidism & $17(4.7)$ & $40(4.8)$ & NS \\
\hline Lung disease & $37(10.2)$ & $55(6.6)$ & .028 \\
\hline Mentally competent & $71(19.7)$ & $240(28.7)$ & .001 \\
\hline Parkinsons & $21(5.8)$ & $49(5.9)$ & NS \\
\hline Renal disease & $16(4.4)$ & $17(2.0)$ & .020 \\
\hline
\end{tabular}

NS $=$ not statistically different, $P>.05$. 
Table 2 shows the distribution of study population characteristics by influenza vaccination status. Men were slightly, but not significantly, more likely to be vaccinated than women. There were no significant differences in vaccination status by age category. Nursing home residents who were reported to have dementia $\langle\mathrm{OR}=1.90(95 \% \mathrm{CI}, 1.47-2.45))$ or Parkinson's disease (OR $=2.31(95 \% \mathrm{CI}, 1.22-4.37)$ ) were significantly more likely to be vaccinated than those without these conditions. Nursing home residents with renal disease $(\mathrm{OR}=.48(95 \% \mathrm{CI}, .25-.96))$, lung disease $(\mathrm{OR}=.59,(95 \%$ $\mathrm{CI}, .38-.91)$ ), heart disease (OR $=.75(95 \% \mathrm{CI}, .59-.97)$ ), and those residents competent to give consent for their own treatment $(\mathrm{OR}=.75(95 \% \mathrm{CI}, .57-.99))$ were significantly less likely to have received influenza vaccine. Residents whose medical records indicated that they had received pneumococcal vaccine were significantly more likely to have also received influenza vaccine (OR $=2.48(95 \% \mathrm{CI}, 1.63-3.77))$.

\section{Influenza Vaccine Effectiveness}

Tables 3 and 4 show the results of two different logistic regression analyses used to estimate the effectiveness of influenza vaccination in reducing the likelihood of influenza-like illness among residents while adjusting for the effects of nursing home and resident characteristics. Table 3 presents the results of the conditional logistic regression. In this model, the odds ratio of .58 indicates a significant reduction

Table 2. Characteristics of 1989-1990 Nursing Home Study Population by Influenza Vaccination Status. South-central and Southwestern Lower Michigan

\begin{tabular}{lccc}
\hline $\begin{array}{c}\text { Influenza Vaccination } \\
\text { Characteristic }\end{array}$ & $\begin{array}{c}\text { Yes } \\
\mathrm{n}=848\end{array}$ & $\mathrm{n}=350$ & $P$ Value \\
\hline Gender & & & \\
Female & $663(78.2)$ & $289(82.6)$ & NS \\
Male & $185(21.8)$ & $61(17.4)$ & \\
Age Category & & & \\
$65-69$ & $45(5.3)$ & $22(6.3)$ & NS \\
$70-74$ & $66(7.8)$ & $37(10.6)$ & \\
$75-79$ & $122(14.4)$ & $53(15.1)$ & \\
$80-84$ & $193(22.8)$ & $80(22.9)$ & \\
$85-89$ & $195(23.0)$ & $86(24.6)$ & \\
$90+$ & $227(26.8)$ & $72(20.6)$ & \\
Pneumococcal vaccination & & & \\
Yes & $169(19.9)$ & $39(11.1)$ & $<.001$ \\
No & $180(21.2)$ & $103(29.4)$ & \\
Unknown & $499(58.8)$ & $208(59.4)$ & \\
Comorbid conditions (not & & & \\
mutually exclusive) & & & \\
Anemia & $52(6.1)$ & $25(7.1)$ & NS \\
Cancer & $17(2.0)$ & $12(3.4)$ & NS \\
Dementia & $419(49.4)$ & $119(34.0)$ & $<.001$ \\
Diabetes & $105(12.4)$ & $46(13.1)$ & NS \\
Heart disease & $455(53.7)$ & $212(60.6)$ & .028 \\
Hypothyroidism & $39(4.6)$ & $18(5.1)$ & NS \\
Lung disease & $55(6.5)$ & $37(10.6)$ & .016 \\
Mentally competent & $206(24.3)$ & $105(30.0)$ & .040 \\
Parkinsons & $59(7.0)$ & $11(3.1)$ & .010 \\
Renal disease & $18(2.1)$ & $15(4.3)$ & .038 \\
\hline N & & & \\
\hline
\end{tabular}

NS $=$ not statistically different, $P>.05$.
Table 3. Conditional Logistic Regression Analysis of Influenza Vaccine Effectiveness in Preventing ILI in Nursing Homes with Proven Introduction of Influenza. 1989-1990 South-central and Southwest Lower Michigan

\begin{tabular}{lrcl}
\hline \multicolumn{1}{c}{ Characteristic } & $P$ Value & Odds Ratio & $95 \% \mathrm{Cl}$ \\
\hline Influenza vaccination & $<.001$ & .58 & $.43-.78$ \\
$\quad$ 1989-90 & & & \\
Lung disease & .059 & 1.61 & $.98-2.63$ \\
Mental competence & $<.001$ & .56 & $.39-.76$ \\
Renal disease & .079 & 1.94 & $.93-4.07$ \\
\hline
\end{tabular}

Imputed influenza vaccine effectiveness estimate $1-O R .1-.58=42 \%$,

Analysis adjusted for sex, age category, and conditional on individual nursing home.

Analysis based on a sample size of $1198: 361$ cases and 837 controls in 23 nursing homes.

Table 4. Logistic Regression Analysis of Influenza Vaccine Effectiveness in Preventing ILI in a Subset of 18 Nursing Homes with Proven Influenza Introduction and Complete Information, Using Overall Percent Vaccinated and Number of Beds to Adjust for the Affect of Nursing Home on the Likelihood of ILI. 19891990 South-central and Southwestern Lower Michigan

\begin{tabular}{lccc}
\hline \multicolumn{1}{c}{ Characteristic } & $P$ Value & Odds Ratio & $95 \% \mathrm{Cl}$ \\
\hline Influenza vaccination & $<.001$ & .58 & $.42-.79$ \\
$\quad 1989-90$ & & & \\
Lung disease & .010 & 2.01 & $1.18-3.42$ \\
Mental competence & .003 & .59 & $.42-.84$ \\
Renal disease & .160 & 1.77 & $.80-3.90$ \\
Percent vaccinated & .069 & .73 & $.52-1.03$ \\
Bed size & .665 & .93 & $.66-1.30$ \\
\hline
\end{tabular}

Imputed influenza vaccine effectiveness estimate 1-OR. 1-.58 $=42 \%$.

Analysis adjusted for sex and age category.

Analysis based on a sample sizc of $922: 282$ cases and 640 controls in 18 nursing homes.

for vaccinated individuals in the likelihood of having an influenza-like illness during the surveillance period $(95 \% \mathrm{CI}$, $.43-.78, P<.001$, imputed vaccine effectiveness estimate of $42 \%$ ). Resident competency, but not lung or renal disease, remained significantly associated with the outcome in this model. This analysis was adjusted for sex and age category and was conditional on individual nursing home.

Table 4 presents the results of the logistic regression analysis that considers the effect of potentially important nursing home characteristics on the likelihood of influenzalike illness among residents. The odds ratio of .58 associated with influenza vaccination again corresponds to a reduction in the odds of developing ILI among the vaccinated $195 \% \mathrm{CI}$, $.42-.79, P<.001$, imputed vaccine effectiveness estimate of $42 \%)$. In this model, lung disease and resident competency were associated significantly with the outcome. Although nursing home size did not have an impact on the likelihood of influenza-like illness among residents, overall vaccination levels did. This effect, although not statistically significant, suggested reduction in the likelihood of illness among residents of homes with higher overall vaccination levels. This analysis included only cases and controls from the nursing homes with information on overall percent vaccinated. When the original conditional logistic regression model was applied 
to this subset population of 18 nursing homes, the odds ratio associated with influenza vaccination was estimated at .64 $(P=.007$, imputed vaccine effectiveness estimate of $36 \%)$.

Vaccine effectiveness may be lower in older people because of a number of factors, and extreme old age may be a surrogate for these factors. We evaluated vaccine effectiveness in two subsets of nursing home residents based on age group. The study population was divided into two approximately equal subsets of residents: those 65 to 84 years old and those older than 84 years. The proportion of individuals who had received influenza vaccine was not significantly different between those aged 65 to 84 years and those more than 84 years old $(69 \%$ vs $73 \%$, respectively, $P=.15)$. Table 5 shows the results of the conditional logistic regression on the younger subset. Influenza vaccination was associated with a significant reduction in the odds of ILI in the younger age group $(\mathrm{OR}=.56(95 \% \mathrm{CI}, .37-.84), P=.005$, imputed vaccine effectiveness estimate of $44 \%$ ). Table 6 shows identical analyses on the subset older than 84 years and although the analysis suggests reduced odds of ILI among the vaccinated subjects, the estimate was not statistically significant (OR $=.65(95 \% \mathrm{CI}, .42-1.01), P=.053$, imputed vaccine effectiveness estimate of $35 \%$ ).

When cases of all ages were examined, it was found that $75 \%$ were treated with antibiotics during their illness, although only 36 cases $(10 \%)$ were identified as having pneumonia in association with their influenza-like illness. Amantadine was used therapeutically in 70 cases in eight nursing homes; $90 \%$ of the treated cases resided in three homes. Thirty-three residents (9\%) died within 1 month of influenzalike illness onset and were considered to have died as a result of influenza-related complications; overall, 45 cases $(12 \%)$ died during the surveillance period. There were too few individuals who had influenza-related pneumonia or who died to evaluate the effectiveness of influenza vaccination in reducing the likelihood of these events.

\section{DISCUSSION}

Although inactivated influenza vaccines have been known for many years to be effective in preventing illness in young adults, the valuc of the vaccine in older individuals has continued to be questioned. ${ }^{2-4}$ For older persons living in the community, studies reported during the period 1992-1995 using different methods in different popula-

Table 5. Conditional Logistic Regression Analysis of Influenza Vaccine Effectiveness in Preventing ILI in a Subset of Nursing Home Residents <85 Years Old, Living in Nursing Homes with Proven Introduction of Influenza. 1989-1990 South-central and Southwestern Lower Michigan

\begin{tabular}{lcccc}
\hline \multicolumn{1}{c}{ Condition } & $P$ Value & Odds Ratio & \multicolumn{2}{c}{$95 \% \mathrm{Cl}$} \\
\hline Influenza vaccination & .005 & .56 & .37 & .84 \\
$\quad$ 1989-90 & & & & \\
Lung disease & .616 & 1.17 & .63 & 2.17 \\
Mental competence & .054 & .64 & .41 & 1.01 \\
Renal disease & .185 & 2.11 & .70 & 6.40 \\
\hline
\end{tabular}

Imputed influenza vaccinc effectiveness estimate 1-OR. 1-.56 = 44\%.

Analysis adjusted for sex and conditional on individual nursing home.

Analysis based on a sample size of $618: 181$ cases and 437 controls in 22 nursing homes. The excluded home had no cases in the $<85$ years age group.
Table 6. Conditional Logistic Regression Analysis of Influenza Vaccine Effectiveness in Preventing ILI and Pneumonia in a Subset of Nursing Home Residents $>84$ Years Old, Living in Nursing Homes with Proven Introduction of Influenza. 19891990 South-central and Southwestern Lower Michigan

\begin{tabular}{lrccc}
\hline \multicolumn{1}{c}{ Condition } & $P$ Value & Odds Ratio & \multicolumn{2}{c}{$95 \% \mathrm{Cl}$} \\
\hline Influenza vaccination & .053 & .65 & .42 & 1.01 \\
$\quad$ 1989-90 & & & & \\
Lung disease & .110 & 1.98 & .86 & 4.55 \\
Mental competence & $<.001$ & .42 & .25 & .70 \\
Renal disease & .183 & 2.05 & .71 & 5.86 \\
\hline
\end{tabular}

Imputed influenza vaccine effectiveness estimate 1-OR. 1-.65 $=35 \%$, not significantly different from unity.

Analysis adjusted for sex and conditional on individual nursing home.

Analysis based on a sample size of 580:180 cases and 400 controls in 23 nursing homes.

tions demonstrated that the vaccines were between 31 and $57 \%$ effective in preventing hospitalization for illnesses diagnosed as pneumonia or influenza during the influenza season; in studies using a case control design, the range was 31 to $45 \% .^{5-8}$ In all studies, the likelihood of these diagnoses actually representing illnesses caused by influenza was increased by timing the data collection to coincide with laboratory-defined influenza virus circulation. The cases were not identified by laboratory confirmation but by their having diagnoses considered compatible with influenza or an influenza-related complication. The consistency among these different studies is remarkable because the seasons were sometimes dominated by type $B$ virus, and the exact clinical diagnostic categories considered in the individual studies varied. In all of these studies, there were unknown percentages of cases that were included but did not truly represent influenza-related illness, and this would lower estimates of vaccine effectiveness.

In the current study, the primary outcome was also an epidemiologic one: an influenza-like illness meeting a broad case definition during a period of confirmed community influenza activity. To increase the likelihood that influenza was the cause of illness in persons who met the case definition for ILI in this study, nursing homes were required to have evidence of introduction of the influenza virus to be included. However, because illnesses were not confirmed with influenza virus isolation, it is clear that our epidemiologic definition is not a clinical one and that some cases classified as ILI might not have actually been influenza-related illnesses. ${ }^{18}$ A large number of homes contributed data and thus can be considered to be broadly representative of all those in the region. Odds ratios generated from logistic regression analyses were used to estimate the reduction in the likelihood of ILI among vaccinated residents. Generally, estimates of vaccine effectiveness in preventing a rare condition in case control studies can be derived from the logistic regression models as being equivalent to 1 minus the odds ratios $(1-O R)$. Inasmuch as ILI was not a rare outcome in the course of influenza outbreaks in the nursing homes described here, this derivation may not be directly appropriate. However, this was done to allow comparison with the results of other studies, especially since there is a body of opinion that vicws this derivation to approximate vaccine effectiveness 
in this situation as appropriate. ${ }^{19}$ Such a calculation is always considered appropriate when an entire cohort of eligible subjects is followed.

The proportion of older nursing home residents who were vaccinated in this study was significantly greater than the vaccination levels among older community-dwelling residents reported in a companion study conducted during the same time period. ${ }^{20}$ In addition, the likelihood of these nursing home residents being vaccinated was not associated with the same chronic conditions as those operating in the community. Nursing home residents with heart, lung, and renal disease and those able to give consent for their own treatment were actually significantly less likely to be vaccinated. This observation may be the result of differences in the way promotion of vaccination was carried out and agreement to be vaccinated was obtained in the community compared with nursing homes.

An earlier study of influenza vaccine effectiveness in nursing homes using another study design found that vaccination was more effective in preventing severe outcomes than simple illness. ${ }^{14}$ These comparisons were not possible in the current study because the number of severe events in those homes with documented influenza transmission was relatively small. There was evidence that protection of individual residents was increased indirectly by the vaccination level of the home, that is, by the effect of herd immunity. While not statistically significant in the model presented here, this relationship has been found when the analysis was carried out at the nursing home level in which the effects of preventing introduction and subsequent spread were both measured. ${ }^{15}$ No information was available on the percentage of nursing home staff members who were appropriately vaccinated before the influenza season, although the importance of this factor in preventing introduction of influenza to residents should not be overlooked.

Most interesting was the difference in prevention of illness when the population was dichotomized by age, with reduced effectiveness for influenza vaccination among the very old compared with those less than 85 years of age. It has been thought, with support from immunologic studies, ${ }^{21}$ that outbreaks that occur even in well vaccinated nursing homes are related to the extreme old age of many of the residents. Although age alone may not be directly responsible for lower rates of effectiveness, it could function as a surrogate for immunologic and other factors directly involved. It could be argued, then, that the very old should be among the highest priority groups for improved influenza vaccines, some of which are now under development. Like younger age, competency to give consent for their own medical treatment emerged as a factor suggesting better health status because competent individuals were both less likely to be vaccinated and less likely to be identified as cases.

Of note also is that antibiotics were used in $75 \%$ of the cases even though only $10 \%$ were diagnosed as having pneumonia. Amantadine, the only licensed antiviral agent against influenza at the time of this study, was used much less frequently and only in a small number of homes. This indicates a need to provide information and guidance about the use of rapid viral diagnostic testing and antiviral agents in the control of influenza to those who are responsible for medical care in nursing home facilities. ${ }^{22}$ In particular, antivirals for prophylaxis might be considered an adjunct to vaccination among the oldest residents in appropriate circumstances, given the limitations of the currently available influenza vaccines in these individuals. ${ }^{23,24}$ This would be of special relevance in those years in which the circulating type A strain is different from that in the vaccine. However, vaccine remains the primary control measure against influenza, and efforts should be made to increase its use among both nursing home residents and staff members to confer individual protection as well as indirect protection through herd immunity. As the distribution of vaccine among residents based on chronic medical conditions and other factors did not follow expected patterns in this study, it may be important to consider barriers to vaccine acceptance and to take action to overcome them so that the risk of both introduction and transmission of influenza in nursing homes can be reduced.

\section{REFERENCES}

1. Davenport FM. Control of influenza, symposium on influenza. Med J Aust Spec 1973;Supp1:33-38.

2. Strassburg MA, Greenland S, Sorvillo FJ et al. Influenza in the elderly: Report of an outbreak and a review of vaccine effectiveness reports. Vaccine 1986;4:38-44.

3. Coles FB, Balzano GJ, Morse DL. An outbreak of influenza A(H3N2) in a well immunized nursing home population. J Am Geriatric Soc 1992;40:589592.

4. Drinka PJ, Gravenstein S, Krause P et al. Outbreaks of influenza $A$ and $B$ in a highly immunized nursing home population. J Fam Pract 1997;45:509-514.

5. Foster DA, Talsma A, Furumoto-Dawson A et al. Influenza vaccine cffectiveness in preventing hospitalization for pneumonia in the elderly. Am J Epidemiol 1992;136:296-307.

6. Fedson DS, Wajda A, Nicol JP et al. Clinical effectiveness of influenza vaccine in Manitoba. JAMA 1993;270:1956-1961.

7. Nichol KL, Margolis KL, Wuorenma H, von Sternberg T. The efficacy and cost-effectiveness of vaccination against influenza among elderly persons living in the community. $N$ Engl J Med 1994;331:778-784.

8. Ohmit SE, Monto AS. Influenza vaccine effectiveness in preventing hospitalization among the elderly during influenza type $A$ and type $B$ seasons. Int $J$ Epidemiol 1995;24:1240-1248.

9. Centers for Disease Control and Prevention. Final results: Mcdicare influenza vaccine demonstration - selected states, 1988-1992. MMWR 1993;42: 601-604.

10. Arden NH, Patriarca PA, Kendal AP. Experiences in the use and efficacy of inactivated influenza vaccine in nursing homes. In: Kendal $A P$ and Patriarca PA, eds. Options for the Control of Influcnza. New York: Alan R. Liss, 1986, pp 155-168.

11. Chapman LE, Tipple MA, Schmeltz LM et al. Influenza-United States, 1989-90 and 1990-91 seasons. MMWR CDC Surveillance Summaries 1992;41:35-46.

12. Simonsen L, Clarke MJ, Williamson GD et al. The impact of influenza epidemics on mortality: Introducing a severity index. Am J Public Health 1997; 87:1944-1950.

13. Monto AS, Ohmit SE, Margulies JR, Talsma A. Medical practice-based influenza surveillance: Viral prevalence and assessment of morbidity. Am J Epidemiol 1995;141:502-506.

14. Patriarca PA, Weber JA, Parker RA et al. Efficacy of influenza vaccine in nursing homes: Reduction in illnesses and complications during an influenza A (H3N2) epidemic. JAMA 1985;253:1136-1139.

15. Arden N, Monto AS, Olmmit SE. Vaccine use and the risk of outbreaks in a sample of nursing homes during an influenza epidemic. Am J Public Health 1995;85:399-401.

16. SAS Institute, Inc. SAS Statistical Softwate, release 6.0. Cary, NC: SAS Institute, Inc., 1988.

17. Statistics and Epidemiology Research Corporation. EGRET Statistical Softwarc. Seattle, WA: SERC, Inc., 1988.

18. Nicholson KG, Baker DJ, Farquhar A et al. Acutc upper respiratory tract viral illness and influenza immunization in homes for the elderly. Epidemiol Infect 1990;105:609-618.

19. Rothman KJ, Greenland S. Modern Epidemiology. Philadelphin: LippincottRaven, 1998, pp 110-111.

20. Ohmit SE, Furumoto-Dawson A, Monto AS, Fasano N. Influenza vaccine use among an clderly population in a community intervention. An J Prev Med 1995;11:271-276. 
21. Phair J, Kauffman CA, Bjornson $A$ et al. Failure to respond to influenza vaccine in the aged: Correlation with B-cell number and function. J Lab Clin Med 1978;92:822-828.

22. Gomolin IH, Lcib HB, Arden NH, Sherman FT. Control of influenza outbreaks in the nursing home: Guidelines for diagnosis and management. J Am Geriatr Soc 1995;43:71-74.
23. Monto AS, Ohmit SE, Hornbuckle K, Pearce CL. Safety and efficacy of longterm use of rimantadine for prophylaxis of type $A$ influenza in nursing homes. Antimicrob Agents Chemother 1995;39:2224-2228.

24. Stilianakis NI, Perelson AS, Hayden FG. Emergence of drug resistance during an influenza epidemic: Insights from a mathematical model. J Infect Dis 1998;177:863-873. 